SLAC-PUB-11560

November 2005

\title{
Linac Coherent Light Source- status and prospects
}

\author{
John N. Galayda, Stanford Linear Accelerator Center \\ 2575 Sand Hill Road MS-18, Menlo Park, California, 94025, USA
}

\begin{abstract}
The Linac Coherent Light Source (LCLS) Project will be an x-ray free-electron laser. It is intended to produce pulses of $800-8,000 \mathrm{eV}$ photons. Each pulse, produced with a repetition frequency of up to $120 \mathrm{~Hz}$, will provide $>10^{12}$ photons within a duration of less than 200 femtoseconds. The project employs the last kilometer of the SLAC linac to provide a low-emittance electron beam in the energy range $4-14 \mathrm{GeV}$ to a single undulator. Two experiment halls, located $100 \mathrm{~m}$ and $350 \mathrm{~m}$ from the undulator exit, will house six experiment stations for research in atomic/molecular physics, pumpprobe dynamics of materials and chemical processes, $x$-ray imaging of clusters and complex molecules, and plasma physics. Engineering design activities began in 2003, and the project is to be completed in March 2009. The project design permits straightforward expansion of the LCLS to multiple undulators.
\end{abstract}

Keywords: Free-electron laser, FEL, self-amplified spontaneous emission, SASE, Linac Coherent Light Source, ultrafast science

\section{PROJECT HISTORY}

The scope and goals of the Linac Coherent Light Source have changed considerably since it was first conceived in $1992^{1}$. At this time, alternatives for design goals were considered ${ }^{2}$; $2-4$ nanometers would cover the "water window," permitting single-shot imaging and holography of biological samples. Operation at 0.1 nanometer wavelength was considered feasible if the full energy capability of the SLAC linac could be harnessed. The earliest proposals were predicated on minimum cost and maximal use of Stanford Linear Accelerator Center facilities. For example, the Final Focus Test Beam (FFTB) enclosure was to house the undulator. A single experiment station, set up adjacent to the FFTB, was similarly modest in scope. As interest in the proposal grew and scientific opportunities came into focus ${ }^{3}$, a target operating range of 1.5-0.15 nanometers was chosen, to be achieved using the last kilometer of the SLAC linac and $1 / 3$ of its maximum operating energy ${ }^{4}$. The LCLS Conceptual Design Report ${ }^{5}$ describes the more extensive facilities proposed in 2002, with six experiment stations located in two experiment halls. Meanwhile, The TESLA Linear Collider program at DESY was expanded to include a VUV/soft x-ray FEL in the TESLA Test Facility ${ }^{6}$, and the TESLA Linear Collider concept was expanded to include a large-scale light source facility with ten undulators fed by beams up to $50 \mathrm{GeV}$ extracted from the collider linac ${ }^{7}$. The TESLA concept inspired the LCLS team to anticipate the need for expansion. The layout of the LCLS has been revised to permit straightforward expansion, preserving the facilities of the initial construction.

Beginning in 1999, the U.S. Department of Energy (DOE Office of Science, Basic Energy Sciences Office) provided R\&D funds to develop the LCLS proposal. In 2001, DOE officially accepted the scientific justification for the LCLS, and authorized the preparation of a project proposal. Engineering design of the LCLS began in 2003. In 2005, funds for "long-lead acquisitions" have been provided for the injector linac, undulator magnets and the undulator measurement facility. Large-scale civil construction will begin in 2006. The LCLS will produce first x-rays early in 2008, and will commence operation in March 2009.

\section{PROJECT BUDGET, ORGANIZATION AND PROGRESS TO DATE}

The total estimated cost of the construction project includes engineering design, purchase of hardware, installation, and system checkout. This amounts to $\$ 315 \mathrm{M}$. It is customary to add this amount to the cost of research \& development

Work supported by US Department of Energy contract DE-AC02-76SF00515 
(since 2002), special spare components and the cost of commissioning activities to arrive at the total project cost of the LCLS, \$379M.

The construction project is a collaboration of Argonne National Laboratory, Lawrence Livermore National Laboratory, the University of California at Los Angeles and Stanford Linear Accelerator Center. SLAC has overall responsibility for project management.

It is natural to divide the project into subsystems:

injector linac, constructed by SLAC

main linac/beam transport/beam dump, constructed by SLAC

undulator systems, constructed by Argonne National Laboratory

$\mathrm{x}$-ray transport/optics/diagnostics, constructed by Lawrence Livermore National Lab

experimental endstation systems, and "soft x-ray" atomic physics experiment, constructed by SLAC civil construction, overseen by SLAC

Activities in 2005 have focused on acquisition of the injector system, undulator major components, and undulator measurement facility. Major procurements include the photocathode drive laser for the injector, the magnetized blocks, pole tips and support beams for the undulators, and the magnet measurement and fiducialization system.

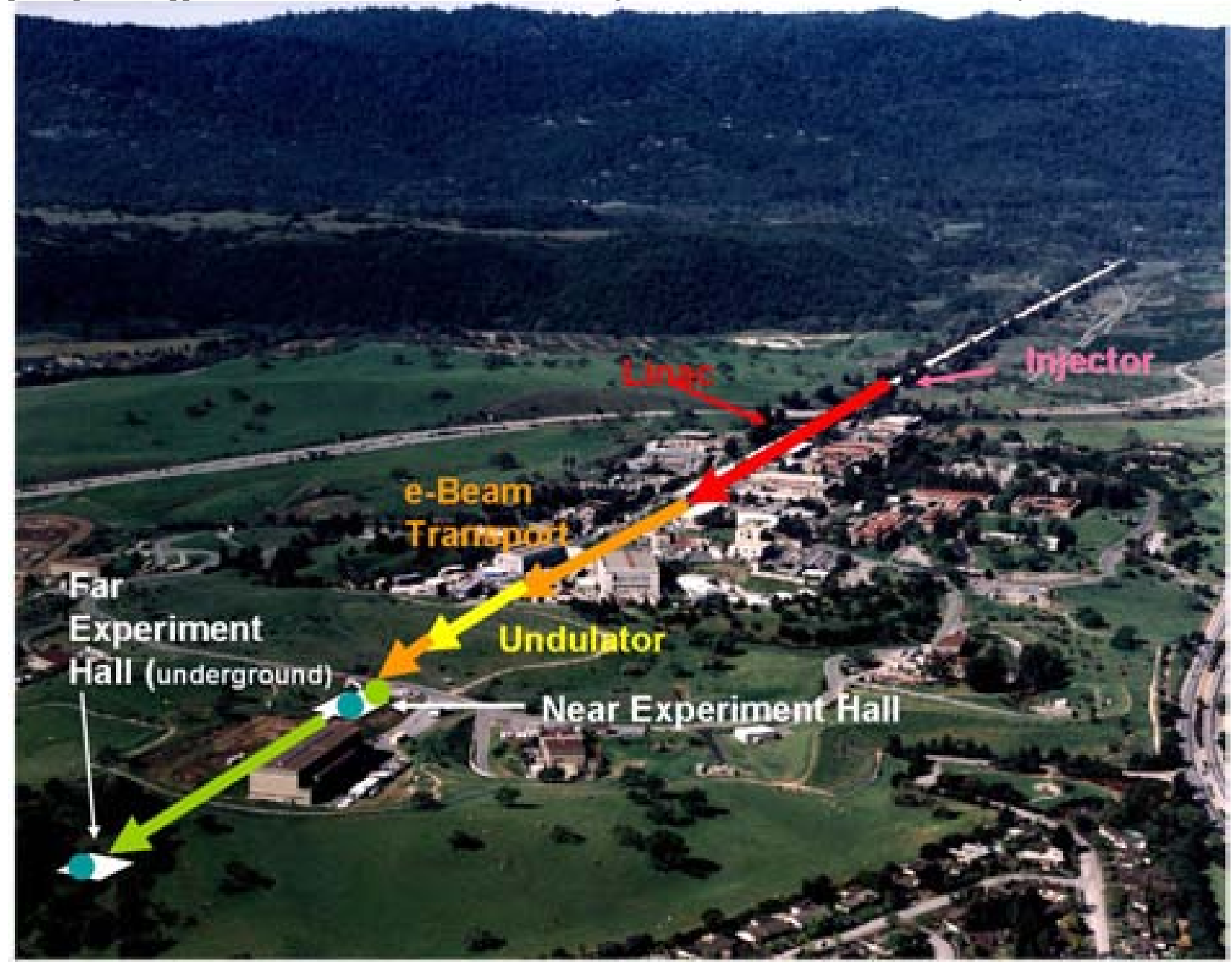

Figure 1. Overview of the SLAC site, showing locations of LCLS facilities. 
In the meantime, the detailed design of accelerator, $\mathrm{x}$-ray experiment and conventional facilities is being completed. First components of the injector linac will be installed in October 2005. Injector installation will be complete by September 2006. Linac and undulator installations will be completed by the end of 2007.

\section{BASIC PERFORMANCE PARAMETERS}

Table 1 lists basic performance goals that guide the design of the LCLS Project. The goals were chosen to be achievable in early operation, and to serve as a basis for planning early-phase experiments. Nominal performance parameters were based on production and acceleration of a 1 nanocoulomb pulse of charge, compressed from 100 amperes and 10 picoseconds to 3,400 amperes and 230 femtoseconds FWHM. Recently it has been determined that $10^{12} 8 \mathrm{keV}$ photons per pulse can be produced from a 0.2 nanocoulomb, 80 femtosecond electron bunch ${ }^{8}$. This low charge operating configuration demonstrates that the LCLS design goals can be reached in a wide range of linac configurations. With $10^{12}$ photons delivered in a single pulse, it will be possible to directly observe the evolution of atomic, molecular and materials properties and transitions with femtosecond-scale temporal resolution.

\begin{tabular}{|c|c|c|c|}
\hline Range of Operation & From: & To: & Units \\
\hline \multicolumn{4}{|l|}{ Photon Beam Parameters } \\
\hline Fundamental radiation wavelength & $\underline{15}$ & 1.5 & $\AA$ \\
\hline Photon energy of fundamental & $\underline{0.82}$ & $\overline{8.2}$ & $\mathrm{keV}$ \\
\hline Coherent photons per pulse & $\underline{10.6}$ & $\underline{1.1}$ & $10^{12}$ Photons/pulse \\
\hline Pulses per sec (linac repetition rate) & 120 & 120 & $\mathrm{~Hz}$ \\
\hline X-Ray pulse duration & 80 & 230 & femtoseconds \\
\hline Peak brightness & $\underline{0.28}$ & $\underline{15}$ & $10^{32} \mathrm{Ph} . / \mathrm{s} / \mathrm{mm}^{2} / \mathrm{mrd}^{2} / .1 \% \mathrm{bw}$ \\
\hline Average brightness & $\underline{\underline{0.16}}$ & $\overline{4.5}$ & $10^{22} \mathrm{Ph} . / \mathrm{s} / \mathrm{mm}^{2} / \mathrm{mrd}^{2} / .1 \% \mathrm{bw}$ \\
\hline Peak saturation power & $\overline{4}$ & $\overline{8}$ & GW \\
\hline FWHM FEL source size & $\underline{116}$ & $\underline{86}$ & $\mu \mathrm{m}$ \\
\hline FWHM FEL source divergence & $\underline{5.7}$ & $\underline{0.8}$ & $\mu \mathrm{rad}$ \\
\hline \multicolumn{4}{|l|}{ Electron Beam Parameters } \\
\hline $\begin{array}{ll}\text { Electron energy } \\
\end{array}$ & 4.313 & $\underline{13.640}$ & $\mathrm{GeV}$ \\
\hline Normalized slice emittance & $\underline{2}$ & $\underline{1.2}$ & $\mu \mathrm{m} \mathrm{rad}$ \\
\hline Charge/bunch & $0.2-1$ & $\overline{0.2-1}$ & $\mathrm{nC}$ \\
\hline Peak current & 1920 & $\underline{3400}$ & $\mathrm{~A}$ \\
\hline FWHM bunch length & 142 & $\overline{25}$ & $\mu \mathrm{m}$ \\
\hline FEL parameter & $\overline{8.5}$ & 4.2 & $10^{-4}$ \\
\hline
\end{tabular}

Table 1. Basic performance parameters of the Linac Coherent Light Source Project

The injector overall configuration has been described in the literature ${ }^{9}$, and nominal performance parameters are shown in Table 2. The injector includes a comprehensive set of diagnostics for measuring emittance and energy spread at the gun output and also at the injector linac output. Slice phase space parameters can be measured by using a transverse deflecting cavity downstream of the injector. The electron beam can be bent onto the main linac axis by the "DL-1" bend (see figure 2.) or, alternatively, directed to a high-sensitivity spectrometer in the main linac tunnel. In the main linac, it will be possible to measure projected and slice phase space parameters prior to passing the beam through the undulator. The bends just upstream of the undulator are designed to provide tight collimation of the electron beam to prevent dark current and other off-energy electrons from entering the undulator system.

The undulator system itself is the subject of a paper in this conference ${ }^{21}$. It includes 33 undulator modules, each 3.4 meters in length. The electron beam is focused by a simple FODO sequence of quadrupoles. The undulator system occupies approximately 131 meters of the 170 meter undulator hall. The beam dump bending magnets occupy about 40 meters downstream of the undulator hall. 


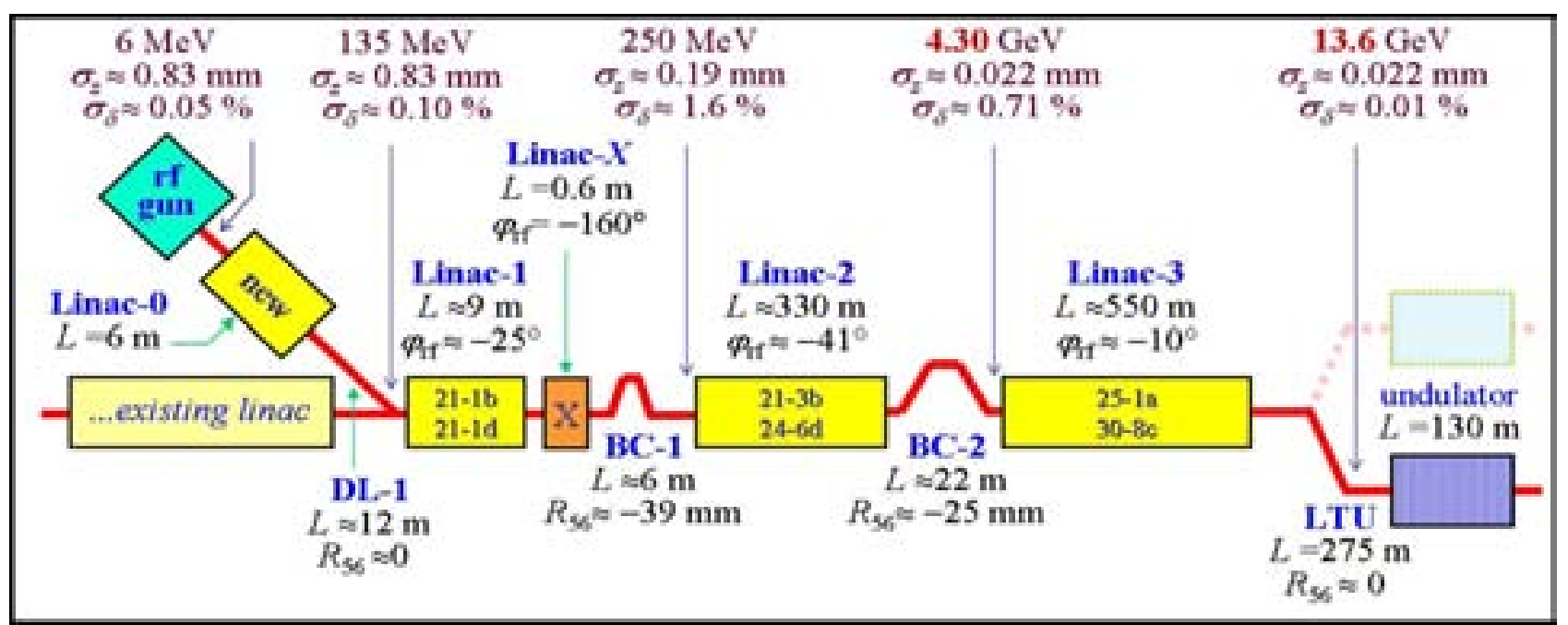

Figure 2. Schematic diagram of LCLS accelerator systems through the undulator. "Linac 0" is the injector linac, located in a vault adjacent to the main linac and just downstream of PEP-II injection systems. "Linac-X" marks the location of the x-band accelerating structure, the purpose of which is to cancel the dependence of electron energy on $\varphi^{2}$, where $\varphi$ is the phase of the bunch centroid with respect to the s-band accelerating field. "BC-1" and "BC-2" mark the locations of the two chicane bunch compressors at $250 \mathrm{MeV}$ and $4.3 \mathrm{GeV}$. "Linac-1", "Linac-2", and "Linac-3" show three segments of the existing SLAC linac with relation to the bunch compressors. "LTU” indicates the Linac-to-Undulator transport line. The displacement of the "undulator" away from the linac centerline indicates that the electron beam is passed through a "dogleg" bend before entering the undulator. The dashed line indicates that a second undulator could be installed in parallel with the first, and could share the linac beam.

\begin{tabular}{|r|c|c|}
\hline Parameter & Value & Units \\
\hline Total length & 14 & $\mathrm{~m}$ \\
\hline electron energy-gun output & 6 & $\mathrm{MeV}$ \\
\hline Initial rms bunch length & 0.83 & $\mathrm{~mm}$ \\
\hline Initial peak current & 100 & $\mathrm{~A}$ \\
\hline Final electron energy & 135 & $\mathrm{MeV}$ \\
\hline Final normalized emittance & 1 & $\mathrm{~mm}$ rad \\
\hline Final rms bunch length & 0.84 & $\mathrm{nC}$ \\
\hline charge & 1.0 & $\mathrm{~A}$ \\
\hline Final peak current & 100 & $\%$ \\
\hline Mean rf phase jitter tolerance & 0.1 & $\%$ \\
\hline Mean rf voltage jitter tolerance & 0.1 & $\%$ \\
\hline
\end{tabular}

Table 2. Nominal injector linac operating parameters

\section{PHOTON BEAM SYSTEMS OVERVIEW}

Downstream of the beam dump is the front-end enclosure (FEE), 35 meters in length, followed by the first experiment hutches in the Near Experiment Hall. The distance from the last undulator to the start of the FEE creates a 79 meter drift path for the x-ray beam. This drift path, added since the publication of the LCLS Conceptual Design Report, is deemed long enough to permit the installation of a pair of offset grazing incidence mirrors that will serve as a low-pass filter to prevent the "hard" component of the spontaneous radiation spectrum from entering the experiment halls. The spontaneous spectrum is quite intense into the $>100 \mathrm{keV}$ range, and would constitute a very troublesome source of background, masking the signal from LCLS experiments ${ }^{10}$. The offset mirrors will be configured like a periscope to displace the first and third harmonics of the LCLS laser beam by $24 \mathrm{~mm}$ from the spontaneous radiation, which is too energetic for specular reflection by the mirrors. Upstream of any optics will be an attenuator system employing a 
nitrogen gas cell for low-energy operation of the FEL (up to about $2 \mathrm{keV}$ ) and a solid attenuator for high-energy operation of the FEL. The front end enclosure will also house the commissioning diagnostics for the FEL.

A wide-field camera observing a scintillator can be inserted directly in the path of the spontaneous radiation or the fully attenuated FEL beam. Calculations of the FEL and spontaneous radiation pattern ${ }^{11}{ }^{12}$ have been used to predict the signal levels detectable with this imaging system ${ }^{13}$. A similar system will be implemented for imaging during operation. This "indirect" imager will use a multilayer mirror to reflect a small fraction of the FEL beam to a scintillator and imaging system. The highly collimated SASE beam will be easily distinguishable from the background spontaneous radiation in these imaging detectors.

For total energy measurements during commissioning, a fast-responding cryogenic calorimeter concept is under development. It is expected to have a measurement/recovery time of about 0.5 millisecond. The calorimeter will provide a coarse image of the x-ray beam to distinguish the FEL beam from background spontaneous radiation. The $\mathrm{x}$-ray beam energy will be deposited in a substrate of silicon or beryllium (for "hard-" or "soft" x-ray operation of the FEL).

Deposited energy will be measured by measuring the power dissipation necessary to maintain a constant temperature in the pixel substrate, as the pixel expels the heat deposited in it as a result of the incident $\mathrm{x}$-ray beam.

Just downstream of the front end enclosure is the Near Experiment Hall (NEH), which will house three experiment hutches for instruments. The hutches are arranged in series; the x-ray beam must pass through the upstream hutches to be used in a downstream hutch. The hutches are $9.5 \mathrm{~m}$ wide by $10 \mathrm{~m}$ along the beam path. The generous width of these hutches will permit the later installation of a second undulator which would deliver x-rays to the Near Experiment Hall while the original LCLS beam is passed through to the Far Experiment Hall (FEH), located 250m beyond. The FEH will house three experiment hutches, $8.5 \mathrm{~m}$ wide by $10 \mathrm{~m}$ long. Grazing incidence mirrors located at the back of the NEH will be used to deflect the LCLS beam along one of three paths separated by about 9 milliradians. In this way it will be possible to deliver beam to any FEH hutch while the other two hutches are open. Initially these mirrors will be re-set on command, to direct $\mathrm{x}$-rays to the desired hutch in the FEH. A longer-range goal is to distribute $\mathrm{x}$-ray pulses to all three hutches at $40 \mathrm{~Hz}$ (1/3 of the LCLS repetition rate) by rapid motion of the mirrors.

The LCLS includes a soft x-ray atomic physics experiment station within the Project scope. It will be placed in the Near Experiment Hall. In addition, a proposa ${ }^{14}$ will be submitted to the Office of Science Basic Energy Sciences program to fund the construction of four sets of experiment instruments for the study of:

X-ray studies of laser-induced transients

Nano-particle and biomolecule imaging

Equilibrium dynamics in condensed matter

Magnetic structures and surface chemistry

A fifth proposal, to create and study plasmas using the LCLS beam, is in preparation for submission to another funding agency.

\section{CONVENTIONAL FACILITIES OVERVIEW}

About $\$ 70 \mathrm{M}$ of the LCLS budget is required for construction of buildings, tunnels and experiment facilities. The injector linac will be housed in a shielded enclosure at the $2 \mathrm{~km}$ point along the $3 \mathrm{~km}$ SLAC linac. This enclosure and another like it at the $1 \mathrm{~km}$ point were part of the original SLAC Linac construction in 1962. A new building must be built above the injector vault, to house the injector laser. From the injector through the linac and beam switchyard, only minor modifications to the existing housing are required. A new radiation enclosure will replace the Final Focus Test Beam Facility. Present plans call for design of the upstream part of this enclosure to permit the addition of five more hard x-ray undulator beam lines. The enclosure, 227m long, will be above ground in the Research Yard. It will connect to the $170 \mathrm{~m}$ undulator hall, located below ground. The underground undulator hall will be tightly controlled in temperature for maximum stability of alignment and magnetic fields in this system. Downstream of the undulator hall is the beam dump area, beyond which (separated by radiation shielding) is the front end enclosure (FEE). After closing an $\mathrm{x}$-ray shutter it will be possible to enter the FEE without requiring the interruption of the electron beam. The Near Experiment Hall will also provide space for timing lasers and experiment staging. Downstream of the NEH, a 250m x- 
ray transport tunnel that will house three simple evacuated x-ray beam transport lines to the Far Experiment Hall. The LCLS facilities have a constant floor elevation; however the terrain of the SLAC site is such that the FEH is about $30 \mathrm{~m}$ below ground. The FEH will be 64.3 meters in length and 14 meters in width, providing ample space around the experiment hutches for laser enclosures, set-up space, etc.

The LCLS Project includes a 78,000 square-foot lab/office building that will house the facility staff as well as Stanford University Ultrafast Science Center ${ }^{15}$. The Center facilities will include six laser lab clusters for research directly related to, or synergistic with, the LCLS.

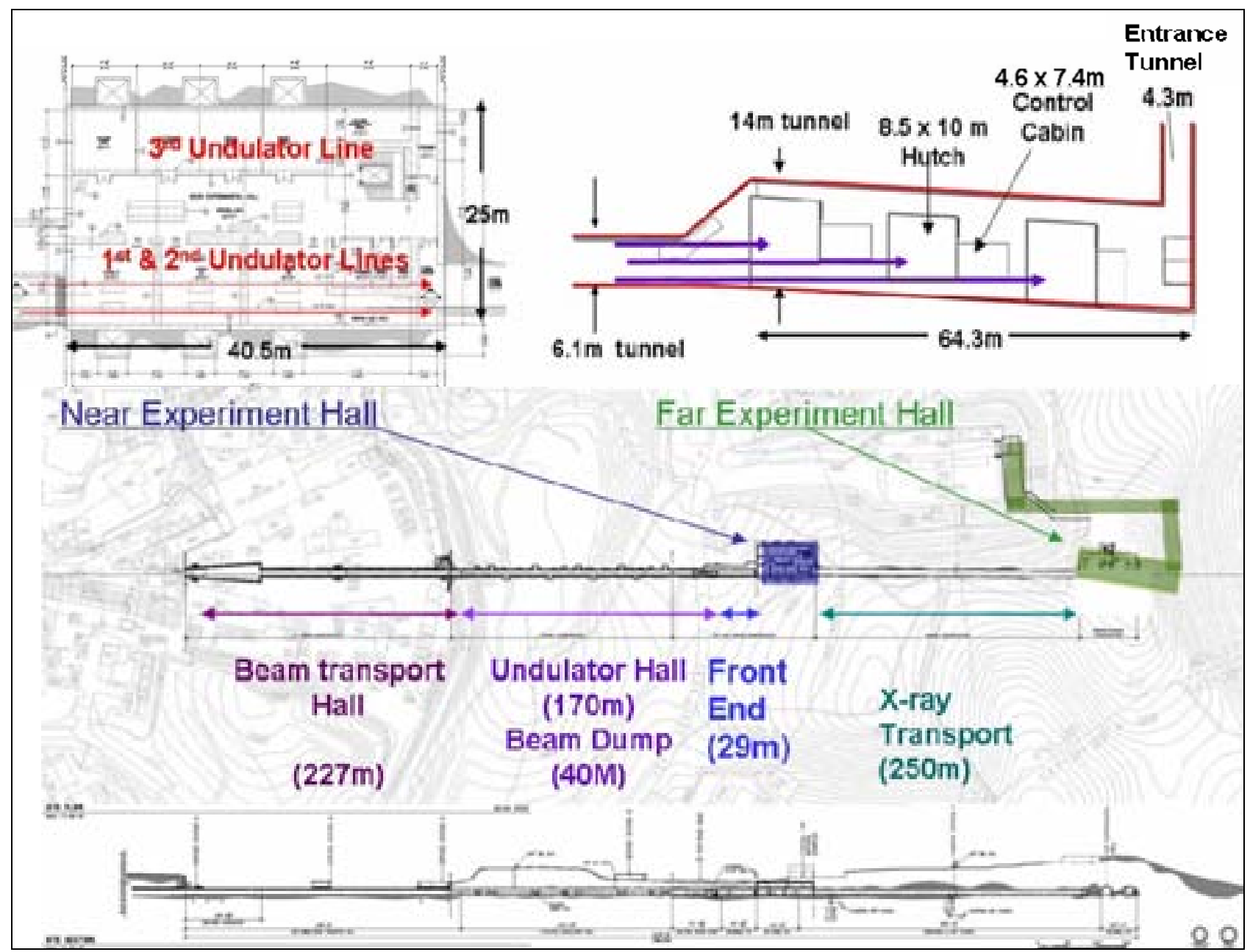

Figure 3. LCLS beam path and experiment station layout

\section{DESIGN CHALLENGES AND SOLUTIONS}

Understanding of the key performance determinants of SASE FELs has improved considerably as knowledge is gained from SASE experiments and FEL design efforts around the world. A discernable pattern of "problem" identification, followed by solution or resolution, emerges from the literature of beam physics as related to SASE; this pattern is easy to see in the bibliography of the LCLS.

The LCLS will begin operation with a gun closely related to the 1.6 cell BNL/SLAC/UCLA design used in successful SASE experiments at the Accelerator Test Facility and Source Development Lab at Brookhaven and the Low Energy 
Undulator Test Line at Argonne National Lab. Significant modifications of the original design will be incorporated in the LCLS gun. It will have dual power feeds ${ }^{16}$, to eliminate any dipole component in the longitudinal accelerating field. The full-length cell will be racetrack-shaped rather than circular, to eliminate the quadrupole component of the accelerating field. For the same reason, dual-feed power input will be incorporated in the first two s-band accelerating structures in the injector linac. In order to operate at $120 \mathrm{~Hz}$, the gun design has added cooling. To minimize the average power dissipated in the gun, a programmed power pulse will be applied to bring the gun to $120 \mathrm{MV} / \mathrm{m}$ as efficiently as possible.

Studies of this design at BNL, ANL and at the Gun Test Facility at SLAC have demonstrated that:

Projected emittance measurements show the summary influence of thermal emittance, intra-bunch variation of betatron match to the focusing channel, deflecting fields in the gun, and space charge effects. Slice emittance measurements are invaluable in order to characterize the distinct contributions of each influence ${ }^{17}$.

In the limit of no space charge effects, the "thermal" emittance of a copper cathode in an electron gun such as that in the LCLS has proven to be approximately twofold larger than simple physical models predict.

Fortunately, further dilution of the transverse phase space of the beam should be controllable so as to meet LCLS goals.

Longitudinal space charge forces, and the dependence of space charge forces on longitudinal and radial position within the electron bunch, are important contributors to longitudinally correlated energy modulation ${ }^{18}$ and incoherent energy spread ${ }^{19}$, respectively.

The effects listed above would, if simply preserved in the acceleration process, have negligible effect on the performance of the LCLS. However, space charge-induced energy modulation of the electrons has detrimental effects on beam quality during the bunch compression process. Space charge forces cause the head and tail of the bunch to be respectively accelerated and decelerated relative to the middle. As a result, the compressed bunch has current spikes at the head and tail. These current spikes produce sufficiently intense wake fields to cause significant energy changes within the bunch as it passes down the undulator channel. The wake fields can enhance SASE in one part of the bunch and degrade SASE in another. The use of wake fields to modulate or shorten the x-ray pulse of the LCLS has been proposed by Reiche and Pellegrini ${ }^{20}$. Because of the very short (100-200 femtosecond) electron bunch length and $5 \mathrm{~mm}$ undulator beam pipe inner diameter, the frequency-dependence of resistivity in the chamber walls must be considered when computing wake fields. ${ }^{21}$ Analysis indicates that, when this AC conductivity effect is taken into account, an aluminum surface produces lower-magnitude wake fields than a copper surface for the LCLS undulator vacuum chamber.

Initial modulation of the electron current as it is produced in the gun will result in a space-charge induced energy modulation. Such current modulation could be produced by intensity fluctuations in the laser pulse striking the photocathode. It is now understood that these fluctuations can be amplified by coherent synchrotron radiation (CSR) forces during the bunch compression process. Theory and simulation indicate that relatively small (6\%) current modulations can be amplified to unacceptable levels by this phenomenon. Theory and simulation show, however, that imposition of a $40 \mathrm{keV}$ energy spread (actually an energy modulation with period $800-1,000 \mathrm{~nm}$ ) in an inverse freeelectron laser will Landau-damp the CSR amplification process, preserving the beam properties desired for SASE ${ }^{22}$.

The design of the LCLS undulator was recognized as a significant challenge when project R\&D began in 1999. A prototype of the 3.4m LCLS undulator module has been built and thoroughly studied by the Advanced Photon Source at Argonne National Lab. The undulator has a fixed gap. The very stringent field tolerance (the undulator parameter $\mathrm{K}$ must be maintained within $0.015 \%$ of nominal) has been met in the prototype. In order to adjust each undulator to its proper $\mathrm{K}$ value, a weak horizontal variation in $\mathrm{K}$ is designed into the magnet. This allows $\mathrm{K}$ to be adjusted by horizontal translation of the undulator with respect to the electron beam trajectory. It will be possible to make this adjustment remotely, during operations ${ }^{24} 25$.

The tolerances on electron trajectory straightness and undulator field are very challenging but achievable with beambased alignment techniques. It has been decided to incorporate electromagnet quadrupoles in the undulator channel which can be used to precisely determine electron beam position with respect to quadrupole centers ${ }^{26}$. Errors in the 
undulator K-parameter that exceed the $0.015 \%$ tolerance can be produced by transverse displacement of one or both ends of the undulator, which cannot be detected solely by their effect on the electron beam. Vertical errors have the tightest tolerance, of the order of 50 microns. The LCLS will be equipped with various diagnostic devices such as beam finder wires and magnet alignment systems to permit alignment of the undulators within the desired tolerance. These devices have excellent sensitivity; however their reliability for absolute alignment depends ultimately on high-precision fiducialization and alignment of critical hardware after installation.

Clearly it would be desirable to have the ability to determine the $\mathrm{K}$ value of an undulator module from observations of the photon beam. Once some SASE gain signal has been detected, it is reasonable to believe that a systematic optimization of FEL power output can be used to direct the alignment of undulators. Since the LCLS undulators have a rather high K value (3.5), a weak SASE signal will be difficult to distinguish from the copious spontaneous radiation. A lock-in detection technique has been proposed to detect weak gain signals. It is based on modulation of the intrinsic energy spread of the electron bunch without affecting any other characteristic ${ }^{27}$.

In principle the spontaneous spectrum from an undulator should contain the information necessary to determine the undulator $\mathrm{K}$ value. This aspect of commissioning an $\mathrm{x}$-ray free-electron laser is an area of active study. It has been the subject workshops organized by UCLA ${ }^{28}$ and LCLS, and by DESY and LCLS under the auspices of the ICFA Future Light Sources Subpanel ${ }^{29}$.

A single-shot spectrometer will be a very powerful diagnostic for the FEL, if sufficient sensitivity and resolution can be achieved. For spontaneous emission, it could be used to verify the correct setting of the undulator K-value. For the FEL signal, it could be used to infer the pulse duration. A serious challenge to this technique comes from the very small emittance and hence small opening angle of the electron beam. For x-rays of any specific energy, a detectable fraction of a large-emittance beam can be expected to satisfy the Bragg scattering condition. However, in the case of the LCLS the small-emittance beam might simply fail to satisfy the Bragg condition of a spectrometer over part of its intrinsic spectrum. Powder diffraction, bent crystals and other concepts are under consideration for a commissioning spectrometer.

An essential part of many LCLS experiments will be the measurement of duration and timing of the pulse of SASE radiation with respect to a pump or timing laser signal. The LCLS will take advantage of techniques developed at the Sub-Picosecond Pulse Source (SPPS) ${ }^{30}{ }^{31}$ for measuring the timing and duration of an electron bunch and x-ray pulse. An electro-optic technique has been used to measure the electron bunch length, with resolution of 270 femtoseconds. The duration of the x-ray pulse from the SPPS was directly measured by a laser pump/x-ray probe measurement of nonthermal melting in InSb. The same laser pulse was used for both measurements. The two measurements of timing (electron bunch with respect to laser, and x-ray pulse with respect to laser) agreed to within 60 femtoseconds. The measurements also show the summary short-term timing jitter of beam delivered from the SLAC damping ring and accelerated to $28 \mathrm{GeV}$ (twice the LCLS energy) is about $200 \pm 60$ femtoseconds. These results compare favorably to the start-up goals for the LCLS. Furthermore the LCLS operating configuration can be expected to produce an improvement in timing stability.

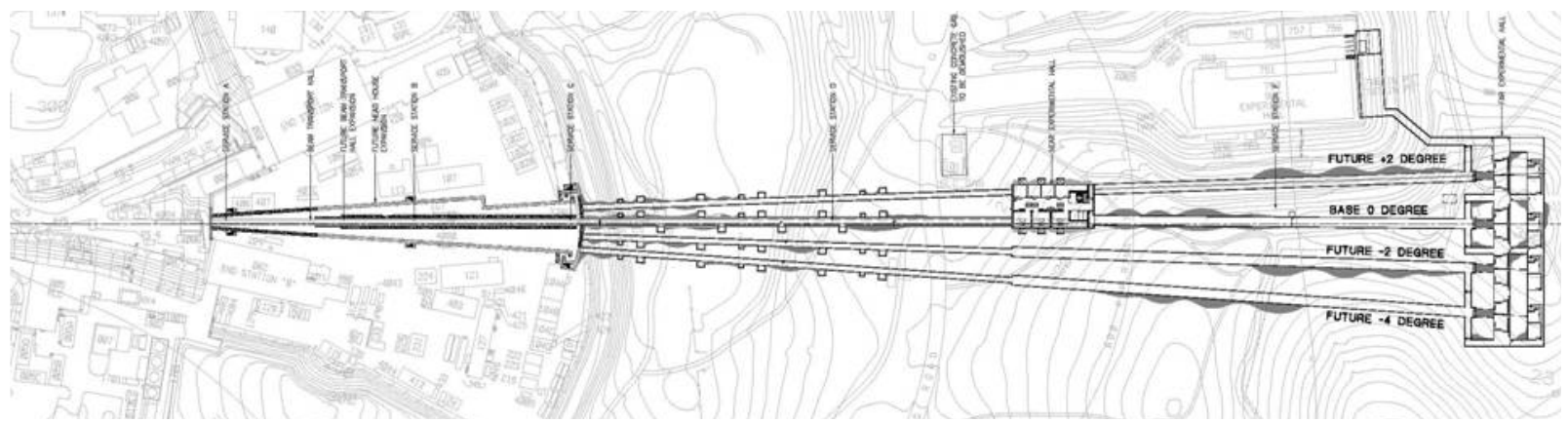

Figure 4. Topological map of the SLAC site showing plan and elevation views of the Linac Coherent Light Source conventional facilities. The figure shows the LCLS Project scope, labeled "base 0 degree" tunnel, sized to accommodate two undulators. Electron optics for four more "hard $\mathrm{x}$-ray" quality lines have been designed, angled at \pm 2 and \pm 4 degrees with respect to the LCLS undulator hall; the \pm 2 degree and -4 degree lines are shown here. 


\section{FUTURE PROSPECTS}

The LCLS will bring researchers to the frontiers of atomic physics, chemistry, materials, fluids, plasmas and molecular biology, with high likelihood for breakthrough discoveries in each of these disciplines. Employment of the SLAC linac and associated facilities provides the most direct, expedient and cost-effective way to begin scientific research with an $\mathrm{x}$-ray FEL. As the LCLS science program matures, the expansion of the capabilities and capacity of the LCLS beyond its initial configuration will follow a natural evolution. As experience with operations is accumulated, it will be possible to implement fast switching of a single $x$-ray beam to several endstations, allowing simultaneous experiments. Experimental evidence and simulation indicate that x-ray pulses in the range of 10-100 fs can be achieved by careful tuning of the LCLS, rather than by hardware modification.

A simple and elegant technique for producing pulses in the range of 1 femtosecond and below has been proposed ${ }^{32}$, which is easily and cheaply adapted to the LCLS. The technique involves the controlled degradation of emittance in the head and tail of each bunch as it passes through a thin slotted foil at the high-dispersion point of the chicane bunch compressor. Only that part of the bunch passing through the slot will have emittance small enough for SASE. Subfemtosecond $\mathrm{x}$-ray pulses should be attainable in this way.

Zholents ${ }^{33}$ has proposed a technique for producing $\sim 1$ micron long, $>10,000$ ampere current spikes within the LCLS bunch. The spikes are produced when a laser pulse is made to overlap and interact with the LCLS electron bunch in a special wiggler magnet just downstream of LCLS Bunch Compressor 2. The interaction could be controlled to produce single 200 attosecond $x$-ray pulses in the LCLS output. Whereas the slotted-foil technique preserves peak SASE power, this "enhanced SASE" (ESASE) scheme will increase peak power considerably within the shortened x-ray pulse. This high peak current could also enable SASE at wavelengths much shorter than $8 \mathrm{keV}$ with an appropriately designed undulator of reasonable length.

Beyond these improvements, a very wide range of options for expanding the capabilities of the LCLS has already been considered $^{34}$, including SASE at $40 \mathrm{keV}$ and beyond, extremely high power at wavelengths longer than $1.5 \mathrm{~nm}$, and self-seeding. Experience in commissioning and operation of the LCLS will enable the identification of the most promising options for upgrade. Of course, demand for a broader range of capabilities will create a need for additional undulator beamlines and experiment stations.

The LCLS is designed to meet future need for expansion. The first step in an expansion will likely be the addition of a second undulator alongside the first. This can be accomplished so as to preserve the initial investment in hardware and civil construction associated with the LCLS baseline project. X-rays from the second undulator could be made available in the Near Experiment Hall while the original LCLS undulator provides x-rays to the Far Experiment Hall.

Layouts have been developed (see Figure 4) for expansion to six undulators, each capable of supporting a "hard” x-ray FEL source and several experiment stations. This expansion would require the construction of four additional undulator halls, fanning out to \pm 2 degrees and \pm 4 degrees in relation to the " 0 degree" undulator hall of the LCLS Project. The Near Experiment Hall is placed to intercept the +2 degree line, providing space for its experiment stations. These bend angles were chosen to make use of transport line components identical to those used for the LCLS Project which were designed to minimize coherent synchrotron radiation effects. The larger bend angles are realized by reversing the sign of selected bend magnets. "Soft" $x$-ray sources do not place as stringent demands on the quality of the electron beam, and could be implemented either by re-use of the electron beams downstream of a "hard" x-ray undulator or by construction of new beam paths outside the \pm 4 degree fan shown in Figure 4.

Electron beams could be distributed to these undulators by the LCLS linac. This distribution could be achieved by AC magnets that, for example, provide pulses to six undulators at 120/6=20 Hz. Alternatively the SLAC linac can accelerate many bunches per linac pulse; indeed it has produced average electron beam powers of nearly $500 \mathrm{~kW}$. These bursts of bunches could be delivered to a single experiment station or else distributed to several stations using RF deflectors. Thus each undulator source would operate at $120 \mathrm{~Hz}$. 
When the PEP-II B-Factory run is completed in 2008, the upstream portion of the SLAC linac will become available for the expansion of the LCLS. The SLAC linac has been designed from the outset to function as three independent linacs. It is already capable of running as a single $50 \mathrm{GeV}$ linac, three independent $14 \mathrm{GeV}$ linacs each running at 120 $\mathrm{Hz}$, or two ( $28 \mathrm{GeV}$ and $14 \mathrm{GeV}$ ) $120 \mathrm{~Hz}$ linacs. This flexibility and energy reach can support the development of advanced FELs for decades to come.

\footnotetext{
${ }^{1}$ Pellegrini, C., "A 4 to 0.1 FEL based on the SLAC Linac", in the proceedings of the Workshop on

Fourth Generation Light Sources, Cornacchia \& Winick, editors, February 24-27 1992, SSRL-92-02, pp. 364-375, 1992

${ }^{2}$ C. Pellegrini, et al., “A 2-4 nm High-Power FEL on the SLAC Linac”, SLAC-PUB-5948, September 1992,

http://www.slac.stanford.edu/cgi-wrap/pubpage?slac-pub-5948 ; Nuc. Inst. Meth A331, pp. 223-227 (1993)

${ }^{3}$ G. K. Shenoy and J. Stohr, editors, LCLS : The First Experiments, SLAC-R-611, September 2000;

http://www.slac.stanford.edu/pubs/slacreports/slac-r-611.html

${ }^{4}$ J. Arthur, et al., LCLS Design Study Report, SLAC-R-0521, 1998

${ }^{5}$ J. Arthur, et al., Linac Coherent Light Source (LCLS) Conceptual Design Report, SLAC-R-593, April 2002;

http://www.slac.stanford.edu/pubs/slacreports/slac-r-593.html

${ }^{6}$ S. Schreiber, et al., "First Lasing at $32 \mathrm{~nm}$ of the VUV-FEL at DESY", Proceedings of the 2005 Free Electron Laser Conference, Stanford University, 21-26 August 2005; to be published electronically at http://www.jacow.org

${ }^{7}$ R. Brinkmann, et al., “An x-ray FEL laboratory as part of a linear collider design”, Nuc. Inst. Meth. A393, pp. 86-92, 1997

${ }^{8}$ P. Emma, et al., “An optimized low-charge configuration of the Linac Coherent Light Source”, SLAC-PUB-11243, May 2005,
} http://www.slac.stanford.edu/pubs/slacpubs/11000/slac-pub-11243.html

${ }^{9}$ D. Dowell, et al., "The Linac Coherent Light Source Photo-Injector overview and some design details”, SLAC-PUB-10765, http://www.slac.stanford.edu/pubs/slacpubs/10000/slac-pub-10765.html also published in the proceedings of the 2004 European Particle Accelerator Conference, Lucerne, Switzerland, 5-9 July 2004, available at the Joint Accelerator Conference (http://www.JACOW.org) website, http://accelconf.web.cern.ch/AccelConf/e04/PAPERS/MOPKF079.PDF

${ }^{10}$ S. H. Rokni, et al., "Radiation safety aspects of the Linac Coherent Light Source Project at SLAC”, SLAC-PUB-11089, July 2005; http://www.slac.stanford.edu/pubs/slacpubs/11000/slac-pub-11089.html ; presented at the

3rd International Workshop On Radiation Safety Of Synchrotron Radiation Sources, 17-19 Nov 2004, SPring8, Mikazauki, Hyogo, Japan; figure 3, page 14 shows the expected flux of hard synchrotron radiation.

${ }^{11}$ S. Reiche, "Spontaneous radiation background calculation for the LCLS", presented at the 2004 Free-Electron Laser Conference, Trieste, Italy, 29 August - 3 September 2004. published at the Joint Accelerator Conference (http://www.jacow.org ) website, http://accelconf.web.cern.ch/AccelConf/f04/papers/MOPOS60/MOPOS60.PDF

${ }^{12}$ R.Tatchyn, "An overview of near-field vs. far-field radiation characteristics of the Linac Coherent Light Source (LCLS)”, SLACTN-05-046, February 2005; http://www.slac.stanford.edu/pubs/slactns/slac-tn-05-046.html

${ }^{13}$ Richard M. Bionta, “X-ray transport optics and diagnostics commissioning report”, UCRL-PROC-207494, October 2004; presented at the LCLS Diagnostics and Commissioning Workshop, 27-28 September 2004, Menlo Park, California;

http://www.llnl.gov/tid/lof/documents/pdf/313158.pdf

14 http://www-ssrl.slac.stanford.edu/lcls/lusi

${ }^{15}$ http://www-ssrl.slac.stanford.edu/ultrafast

${ }^{16}$ L. Xiao, et al., "Dual feed RF gun for the LCLS”, SLAC-PUB-11213, May 2005, http://www.slac.stanford.edu/pubs/slacpubs/11000/slac-pub-11213.html ; to be published in the proceedings of the 2005 Particle Accelerator Conference, 16-20 May 2005, Knoxville, TN; also to be published at the Joint Accelerator Conference Website ( http://www.JACOW.org )

${ }^{17}$ D. H. Dowell, et al., "Slice emittance measurements at the SLAC gun test facility”, SLAC-PUB-9540, September 2002, http://www.slac.stanford.edu/cgi-wrap/pubpage?slac-pub-9540 ; also published in Nucl. Inst. Meth. A507, pp 327-330 (2003)

${ }_{18}^{18}$ T. Shaftan, et al., Experiments with electron beam modulation at the DUVFEL accelerator”, SLAC-PUB-10858,

2004, http://www.slac.stanford.edu/pubs/slacpubs/10000/slac-pub-10858.html , also published in Nuc., Inst. Meth. A528, pp. 397401, 2004

${ }^{19}$ Z. Huang, et al., "Uncorrelated energy spread and longitudinal emittance of a photoinjector beam”, SLAC-PUB-11240, May 2005, http://www.slac.stanford.edu/pubs/slacpubs/11000/slac-pub-11240.html; contributed to the 2005 Particle Accelerator Conference, May 2005, Knoxville, TN.

${ }^{20}$ S. Reiche, et al., "Pulse length control in an X-ray FEL by using wakefields", published in the proceedings of the 2003 Particle Accelerator Conference, 12-16 May 2003, Portland, Oregon; published at the Joint Accelerator Conference ( http://www.jacow.org ) website, http://accelconf.web.cern.ch/accelconf/p03/PAPERS/TOAC010.PDF

${ }^{21}$ K. L. F. Bane and G. Stupakov, "Resistive wall wake field in the LCLS undulator," SLAC-PUB-11227, May 2005, http://www.slac.stanford.edu/pubs/slacpubs/11000/slac-pub-11227.html, also to be published in the proceedings of the 2005 Particle Accelerator Conference, 16-20 May 2005, Knoxville, Tennessee; to be published at the Joint Accelerator Conference Website, http://www.jacow.org 
${ }^{22}$ C. Limborg-Deprey, et al., “Computation of the longitudinal space charge effect in photoinjectors,”, SLAC-PUB-11170, July 2004; http://www.slac.stanford.edu/pubs/slacpubs/11000/slac-pub-11170.html ; published in the proceedings of EPAC 2004, 5-9 July 2004, Lucerne, Switzerland; also available at the Joint Accelerator Conference Website:

http://accelconf.web.cern.ch/AccelConf/e04/PAPERS/TUPLT162.PDF

${ }^{23}$ L. D. Bentson, et al., "Inverse free electron laser heater for the LCLS", SLAC-PUB-11170, July 2004;

http://www.slac.stanford.edu/pubs/slacpubs/11000/slac-pub-11186.html ; published in the proceedings of EPAC 2004, 5-9 July

2004, Lucerne, Switzerland; also available at the Joint Accelerator Conference (http://www.JACOW.org) Website:

http://accelconf.web.cern.ch/AccelConf/e04/PAPERS/MOPKF083.PDF

${ }^{24}$ I. Vasserman, et al., "LCLS undulator design development”, published in the proceedings of the 2004 FEL Conference, 29 August

- 3 September 2004, Trieste, Italy; published at the Joint Accelerator Conference (http://www.JACOW.org) Website:

http://accelconf.web.cern.ch/AccelConf/f04/papers/TUBOS04/TUBOS04.PDF

${ }^{25}$ S. Milton, “The Linac Coherent Light Source and undulator system”, these proceedings

${ }^{26}$ P. Emma, et al., "Electro-magnetic quadrupole magnets in the LCLS undulator”, SLAC-TN-05-016, http://www.slac.stanford.edu/pubs/slactns/slac-tn-05-016.html

${ }^{27}$ P. Emma, et al., "Weak FEL gain detection with a modulated laser-based beam heater”, SLAC-PUB-11242, http://www.slac.stanford.edu/pubs/slacpubs/11000/slac-pub-11242.html , also to be published in the proceedings of the 2005 Particle Accelerator Conference, 16-20 May 2005, Knoxville, TN; to be published at the Joint Accelerator Conference Website ( http://www,JACOW.org )

${ }^{28}$ R. Bionta, et al., "Report of the LCLS Diagnostics and Commissioning Workshop, Los Angeles, USA, January 19-20 2004”, SLAC-R-715; http://www.slac.stanford.edu/pubs/slacreports/slac-r-715.html

${ }^{29}$ ICFA (Future Light Sources Sub-Panel) Mini Workshop on Commissioning of X-Ray Free-Electron Lasers, April 18-22 2005, DESY-Zeuthen; http://commissioning2005.desy.de/index_eng.html ; a report of the workshop will appear in ICFA newsletter 36 which will appear at http://www-bd.fnal.gov/icfabd/news.html

${ }^{30}$ A.L.Cavalieri, et al., “Clocking femtosecond x-rays”, PRL 94, 114801, 4 pages (2005)

${ }^{31}$ A.M.Lindenberg, et al., “Atomic-scale visualization of inertial dynamics”, Science vol. 308, pp. 392-395, 15 April 2005

${ }^{32}$ P. Emma, et al., "Femtosecond and sub-femtosecond pulses from SASE-based free-electron laser", SLAC-PUB-10002, Mar 2004. 11pp. http://www.slac.stanford.edu/pubs/slacpubs/10000/slac-pub-10002.html ; Published in Phys.Rev.Lett.92:074801,2004

33 A. A. Zholents, "Method of an enhanced self-amplified spontaneous emission for x-ray free-electron lasers", Phys. Rev. Special

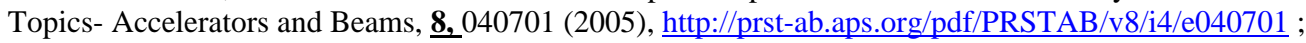

A. A. Zholents, et al., „Current-enhanced SASE using an optical laser and its application to the LCLS”, SLAC-PUB-10713,

September 2004, http://www.slac.stanford.edu/pubs/slacpubs/10000/slac-pub-10713.html , also published in the proceedings of the 26th International Free Electron Laser Conference and 11th FEL User Workshop, Trieste, Italy, 29 Aug - 3 Sep 2004; available at the Joint Accelerator Conference (http://www.JACOW.org ) website, http://accelconf.web.cern.ch/AccelConf/f04/papers/WEBOS01/WEBOS01.PDF

${ }^{34}$ M. Cornacchia, et al., "Future possibilities of the Linac Coherent Light Source", SLAC-PUB-10133, http://www.slac.stanford.edu/pubs/slacpubs/10000/slac-pub-10133.html , published in J. Synchrotron Radiat.11, pp.227-238, 2004 